Revista de Neuro-Psiquiatría, 56: 139-146, 1993

\title{
NARRATIVA Y PSICOTERAPIA
}

\author{
Por EFRAIN A. GOMEZ
}

\begin{abstract}
RESUMEN
El enfoque exclusivo en teoría y en técnica psicoanalítica o psicoterápica, de cualquier tipo, no llega a compensar la lectura continuada y el estudio repetido de los genios clasicos e inmortales de la literatura y del arte universal. Este artículo resalta un nuevo hallazgo de la investigación experimental con niños: la verdad narrada puede ser más importante que la verdad histórica en la promoción de la salud mental. Este es un hallazgo inesperado para los investigadores de la psiquiatría infantil, pero no para los clínicos humanistas que intuyeron por mucho tiempo la importancia de la narrativa in el desarrollo de la mente humana.
\end{abstract}

\section{SUMMARY}

The exclusive focus on theory and technique, psychoanalytic or otherwise, will never compensate the reading and the study of the immortal classics in literature and art. This paper highlights a new finding of research on infants and children: historical true might not be as important as narrative true in the promotion of mental health. This finding was unexpected for the investigators that thought that historical true always predicted behavior. Humanistic psychiatrists always had the intuition of its importance in the develop of the human mind.

Palabras - clave: Psicoterapia, narrativa, investigación infantil, clásicos inmortales.

KEY wORDs: Psychotherapy, narrative, infant research, immortal classics.

En otro artículo nos ocupamos del papel que desempeña el estudio de la literatura clásica en la formación del psicoanalista (GOMEZ, 1986). En ese artículo señalamos que el enfoque exclusivo en la teoría y la técnica psicoanalítica no llega a compensar la lectura continuada y repetida de los genios clásicos de la literatura universal. El entendimiento de cómo la gente ha vivido a través de los siglos y 
cómo ha interpretado sus vivencias, no puede tener otro objeto que enriquecer y ahondar nuestro conocimiento de la condición humana.

Everybody thinks that I stand by the scientific character of my work and that my principal scope lies in curing mental mala-dies. This is a terrible error that has prevailed for years and that I have been unable to set right. I am a scientist by necessity... I am really by nature an artist (Giovanni PAPINI, 1989). Freud continúa su entrevista imaginaria con PAPINI intimando que en su psicoanálisis se puede encontrar la influencia de Heine, Zola y MALLARME bajo el tutelaje todopoderoso de su maestro, GoEThe. Es conocido que Freud siempre fue un ávido lector de los clásicos, y que aprendió el español con el sólo objeto de leer Don Quijote.

Su preocupación intelectual siempre oscilo entre las dos grandes tradiciones de la civilización occidental, la ciencia y las humanidades. Nunca recibio el premio Nobel, pero si el premio Goethe en literatura.

Hace cuatro siglos el Macbeth de SHAKESPEARE le dice al médico en referencia a Lady Macbeth que sufre de ansiedad, remordimiento, sonambulismo y la compulsión de lavarse las manos:

Cure her of that

Canst thou not minister to a mind disease,

Pluck from the memory a rooted sorrow,

Raze out the written troubles of the brain,

And with some sweet oblivious antidote

Cleanse the stuffd bosom of that perilous stuff which weighs upon the heart?

[SHAKESPEARE, 1952, V, iii, p. 308]
Implicada en esta cita encontramos la necesidad de mezclar la medicina con las humanidades. Cure her of that..., Macbeth le dice al médico. Cuando el médico responde Therein the patient must minister to himself, Macbeth responde throw physics (medicine) to the dogs- $I l l$ none of $i t$. ShaKespeare enten- día que se necesitaba más que medicina para arrancar una memoria enraizada en el dolor (pluck from the memory a rooted sorrow), o sacar de raíces los engramas conflictivos del cerebro (the written troubles of the brain). Macbeth habla al mismo tiempo al médico y a Seyton. Al médico le dice que sus nobles lo están abandonando; y luego agrega que aplaudirá con entusiasmo si el médico encontrara una cura para la enfermedad de su nación.

Antes del descubrimiento del psicoanálisis freudiano, los escritores clásicos ya habían hecho uso generoso de los mitos, las leyendas, la metáfora, la alegoría, la paradoja, y la ficción poética para explicar la razón de la sinrazón en asuntos del alma y del corazón del hombre. Para los griegos los mitos eran los organizadores de la mente y las estructuras invisibles del alma. Es más, los mitos no sólo representaban observaciones empíricas sino verdades eternas. Comose puede ver, los griegos percibieron el balance entre logos y mythos. Este significado fué subsecuentemente distorcionado por la religión cristiana cuando fanáticamente trató de demostrar que la religión griega estaba llena de falsedades. Lamentablemente, desde entonces, la palabra mito es sinónimo de falsedad.

La piedra angular del edificio psicoanalítico lleva el nombre de un mito griego clásico, Edipo. No se sabe si Freud estaba consciente que al considerar el 
complejo de Edipo como núcleo de toda neurosis estaba elevando las funciones de la mente a un plano poético. Los griegos consideraron que la narrativa de nuestras vidas, los personajes que encarnamos, $y$ los sueños que soñamos, están estructurados por una logica selectiva y profunda llamada mythos.

La ficción usa la narrativa para explicar las manifestaciones evasivas de la naturaleza humana. La trama demuestra como todos los aspectos de la narrativa se interrelacionan, dándole a la historia sentido y coherencia interna. La historia se relaciona con el qué, cuándo, y cómo; la trama con el porqué.

En nuestro trabajo clínico, la trama se llama teoría, la historia, anamnesis. Sin embargo debemos subrayar que el mito es más que la teoría y más que la trama porque va más allá de la interaccion humana tratando de abarcar larelación con lodivino.

La adquisición del lenguaje hablado incrementa la habilidad del niño para narrar historias. Estas historias o relatos cambian la percepción del niffo con respecto a sí mismo y al mundo que lo rodea. Word knowledge no es lo mismo que world knowledge. Narrativa no es to mismo que descripción pura. Narrativa implica una manera diferente de pensar; sugiere la actuación de personas como agentes de sus propios destinos con intenciones y comportamientos propios. Todo relato debe tener una secuencia causal con un principio, una fase intermedia, $y$ una final, la narrativa es un fenómeno universal en el desarrollo de la mente humana, sus motivaciones y sus significados (STERN, 1985). Esta es un área nueva de investigación que ayudará a clarificar cómo, cuándo y porqué los niños construyen historias. El dominio de la relación verbal del self puede subdividirse en un sentido categorico de un self que observa, objetiviza y etiqueta y un self que es, vive, experimenta, mezclando elementos de otros sentidos y otras dimensiones de la existencia.

Experimentadores, investigando patterns of attachment con el objeto de predecir los resultados de la relación madre-nifio encontraron algo inesperado: la relación madre-niffo no es una simple repetición de la relación madre-niño que le precede; sino que depende de la manera cómo los participantes de esta relación diádica particular, experimentaron e interpretaron dicha relación (STERN, 1989). En otras palabras, lo importante no es lo que le pase a uno, sinó la manera como uno percibe lo que le pasa, como lo interpreta, y cómo lo narra. La coherencia y la viabilidad de la narrativa en el contexto de nuestro presente tiene precedencia sobre el pasado y el futuro. En la experiencia vivencial presente se juntan el presente, el pasado, y el futuro. En el caso particular del artista la conversión de la realidad externa a una realidad ficticia deber ser completa y convincente, para hacerla cré́ble, estética y agradable. La transmutación de la realidad a la fícción se consigue a través la manipulación imaginativa de secuencias temporales especiales de los sistemas estructurales y ontológicos de la mente. El registro de acciones simultáneas pero diferentes en el mismo espacio narrativo, la habilidad de transformar dos diálogos espacial y temporalmente independientes en uno, el paralelismo entre diferentes formas de pensar y sentir la fragmentación o refracción de ocurrencias comunes, son técnicas que cambian niveles de realidad, y ayudan a crear una nueva realidad, a inventar una nueva realidad en la que el lector pasa a formar parte de ella. 
Si las madres tienen experiencias negativas, con sus propias madres, pero son capaces de inventar una narrativa coherente y viable, el pronóstico es bueno. Esto demuestra, en muchos aspectos, que la verdad histórica es menos importante que la verdad narrada.

All the world's a stage,

And all men and women are merely players [SHAKESPEARE, As You Like it].

La analogía entre el teatro y la mente se basa en una analogía más general, la analogía entre el teatro y la vida. Este reconocimiento viene desde Platón y HOBBES, y es subrayado en las obras clásicas de Shakespeare y Cervantes.

En el prólogo de un Fragment of an Analysis of a Case of Hysteria, el caso de Dora es referido como un Roman a clef, como advertencia a los lectores que leerán el caso de Dora no como una contribución a la psicopatología de la neurosis, sinocomonovela picaresca. (FrEUd, 1959). Freud usa un término literario francés, lo quehace pensarque estaba en esemomento, empeñado en la creación de una forma literaria sin precedentes.

El caso de Dora es considerado por algunos como la lliada de la literatura psiquiátrica. Sabemos que la represión y el compromiso forman parte de la fórmula freudiana para explicar los sueños y los síntomas. Esta formula aplicada al trabajo de FrEUD, a principios de siglo, muestra la gestación de un compromiso, en el mismo FrEUD, entre sus afanes de médico y sus afanes de literato. Su estilo revela un contenido expreso que es médico y un contenido tácito que es poético. Es de notar que los casos clínicos de Freud se pueden leer como novelas románticas porque expresan el lado ficticio de la naturaleza humana, su romance y sus pasiones. A The Phobia in a Five-Year-OldBoy y Comments on a Case of Paranoia se les conoce más por sus nombres ficticios que científicos: El niño Juanito y El caso Shreber. Es interesante señalar que FrEUD no analizó directamente ni al niño Juanito ni a Daniel Shreber. Analizo la historia que le narro el padre de Juanito y el libro que Shreber escribio sobre su psicosis y su tratamiento psiquiátrico.

La novela tiene como bases, el relato y la trama. Todo aquello que es observable forma parte del relato. Pero el lado romántico de la naturaleza humana incluyendo sus pasiones, sus sueños, sus goces, sus dolores y todo aquello que la vergüenza impide mencionar es parte de la trama (Foster, 1927). El relato responde a la secuencia de los hechos, el qué, el cuándo y el cómo. La trama revela las intenciones y nos dice el porqué.

La trama en el relato es la teoría en la psicología. Revelar el porque de nuestras acciones fue la intención principal de la interpretación freudiana. La trama principal en la teoría de FreUd es el complejo de Edipo.

Si bien es cierto que la trama y el mito tienen mucho en común, los griegos clásicos nos recuerdan que el mito va más allá que la teoría y la trama, ya que incluye lainteracción de lo humano como lo divino.

Otro aspecto antes no señalado es la relación entre narrativa y el concepto que en alemán se llama Nachtraglichkeit (MODELL, 1990), un término redescubierto por Freud pero ignorado por sus seguidores, hasta hace poco. Este concepto se refiere a la idea del reciclaje periódico de los recuerdos en categorías cognitivas y afectivas nuevas bajo el impacto de experiencias vivenciales contemporáneas.

Este concepto podría dar lugar a una teoría derivativa que sugiere que el desarrollo psicológico se desenvuelve es- 
pontáneamente en ciclos y no en forma linear inalterable.

En su libro Neural Darwinism, Edelman (1987) cambia de enfoque, en vez de concentrar su atención en la memoria del sistema inmunologico, concentrasuatención en las funciones del cerebro con respecto a las funciones de la memoria. EDELMAN piensa que lamemoria no se almacena en forma inalterada y para siempre, sino que continuamente se recategoriza; así por ejemplo, atribuciones retrospectivas con respecto al pasado se revisan en forma cíclica en relación al contexto de experiencias psíquicas subsecuentes. Esto quiere decir que traumas o deficiencias, consideradas en forma aislada, no siempre producen desordenes psiquiátricos. En otras palabras, la ontogenia no siempre es copia carbón de la filogenia.

Desde una perspectiva de sentido y de significados, el cambio de nuestra conducta, de nuestra manera de sentir, o de pensar se produce no en el lugar donde se genera el conflicto o el trauma, sino en otro lugar, en otro dominio, en uno donde se pueda dialogar, narrar historias hasta encontrar una viable. Un dominio conducente a la coexistencia, al apoyo incondicional, y al diálogo circular espontáneo.

Una forma de cambiar nuestros pensamientos, sentimientos y nuestro comportamiento es a través del relato repetido de nuestras vidas. La repetición genera y regenera nuevas significaciones, en ellas empezamos a ver la influencia de diferentes niveles de conciencia. El deseo universal, el querer retomar el poder, delegado, sobre nuestros destinos es una de las motivaciones más poderosas para el cambio que debe operarse en todo ser humano que quiere seguir desarrollándose. En la realización de este deseo el lenguaje y la narrativa juegan papeles importantes.

La historia que traen los pacientes se parecen a cuentos, o a novelas. Como relatos pueden ser simples o pueden ser complejos, superficiales o profundos, coherentes $o$ incoherentes, a veces tienen principio pero no fin, o carecen de principio y de fin, etc. Desde este punto de vista la psicoterapia puede ser breve o interminable.

Comparemos la psicoterapia breve y la interminable con el cuento y la novela respectivamente. El cuento es un arte de revelación moral y de transformación parcial. La novela es un arte de evolución moral y tiende a la transformación total. En el cuento se espera un cambio de consciencia en el protagonista, un cambio de percepción, una autorevelación, una manera diferente de ver las cosas, un trabajo de reparación en la personalidad. En la novela usualmente se ve un cambio de consciencia en el protagonista y los personajes principales, una alteración de su manera de ser, una reconstrucción de su personalidad, un acto de descubrimiento que tiende a promover cambio no sólo en la personalidad del protagonista sino también en el mundo que lo rodea.

Como vemos, la adquisición del lenguaje y la utilización de la palabra en la psicoterapia es esencial. Pero también es cierto que el lenguaje hablado no es sólo unabendición, sino también una maldición. El lenguaje hablado hace que nuestra experiencia preverbal global, se transforme en una experiencia parcial. Expliquémonos, el lenguaje hablado produce una escición entre dos formas de experiencia interpersonal, la experiencia vivida y la experiencia narrada. En el mundo preverbal, el niño goza una experiencia global sin límites. La experiencia preverbal 
es una mezcla de diferentes sensaciones, percepciones y simbolismos únicos.

El advenimiento de la palabra impone la reducción de la experiencia a una, usualmente visual. Supongamos, por ejemplo, que estamos considerando la percepción infantil de un raye de sol que se refleja en la cuna de' un niño. El infante experimenta en forma global la intensidad, el calor, la forma, la brillantez, el placer, la seguridad, el abrigo y otros aspectos del rayo solar. El hecho que sea un rayo de luz no es ni primario ni importante. Para apreciar $y$ mantener esta perspectiva omnidimensional, el infante no presta atención a cualidades secundarias o terciarias como el color. Para el niño es más que una experiencia visual: Supongamos que en ese momento llega la madre y dice "mira, el rayo de luz es amarillo".' Las palabras, en este caso, aislan la experiencia total y la transforman en una experiencia predominantemente vísual (STERN; 1985).

El lenguaje hablado fractura la experiencia global del niffo introduciendo una discontinuidad entre la experiencia verbal y la preverbal. Esta es posiblemente la razón que impela al ser humano a la búsqueda del arte, en todas sus manifestaciones, como canal sublimatorio, para expresar lo que no se puede expresar con palabras. La ciencia y el arte son dos polos de la experiencia humana que se suplementan y complementan.

Con el objeto de senalar la importancia manifiesta y latente de la realidad en lanarrativa presentamos dos viñetas, no de pacientes que sólo son conocidos por sus terapeutas; sino de dos escritores conocidos por todo aquel que se interesa por la literatura universal.

José María Arguedas es uno de los pocos escritores latinoamericanos que ha sido capaz de describir el simbolismo preverbal en su narrativa. Esa etapa preverbal del desarrollo en la que el sentido de un ser emergente se hace presente, tratando de encontrar su esencia. El sentido subjetivo del ser en formación tiende a expresarse en forma global porque la capacidad de la palabra, todavía no ha llegado.

ARguedas esta considerado entre los grandes de la literatura mundial, es el primer peruano, capaz de destilar su poesía del quechua al castellano. ARGUEDAs describe labelleza del paisaje andino impresionante e intimidante al mismo tiempo, y lo utiliza como fondo y forma de su narrativa.

ARGUEDAS narra la aterradora condición social del indio peruano, utilizando la fuerza preverbal avasalladora que sólo una experiencia total puede dar. ARGUEDAS pinta al Pení como hervidero de las más complejas formas de cultura y racismo con exactitud documental, pero sin sacrificar su poesía. El Pení es uno de los países más racistas del planeta, sin embargo no hay libros escritos sobre su racismo sui generis.

Continuando con ARGuedas, el logra instilar en el castellano la frase estructural, el ritmo, y el vocabulario del quechua Aunque es casi imposible comunicar los matices de sus novelas en otros idiomas, Francis Horning Barraclough logra lo imposible en su traducción Deep Rivers (1991). Los Rios Profundos fué publicada en 1958, recibió en 1962 el premio de la novela Iberoamericana, otorgado por la fundación William Faulkner. Nadie como él, ni antes ni después, hanarrado la pureza simple de las valoraciones elementales que funden el subjetivismo del personaje con la realidad objetiva, sin deformarla. Ernesto, comoel mismo ARGUEDAs, es hijo de blancos pero lo amamanta una india, . ARGUEDAs es capaz de sacarle provecho a esta experiencia preverbal. En su novela pinta, con maes- 
tria, las poderosas corrientes que golpean al mino que; solo, tiene que enfrentarse a an mundo poblado por monstruos, fuego, hielo, paisajes terrestres y lunares. Entesto, el narrador, es un nifo con origenes en dos mundos opnestos. Hijo de un abogado de pueblo, vagabundo. Es ctiado por sirvientes indios hasta los catorce affos cuando entra a una escrela católica como pupilo: En este ambiente urbano Enesto es un inadaptádo y un introvertido pesional. Uni tema mayor en Los Ríos Projiundos es el vfneulo de Emestó con el mundo natural que lo rodea y a l que le da alina y vìđa; y ai hacerlo vámás alláde la metáfora para convertirla en magia. Ernesto escuicha las voces de los dioses muertos: Los ríos, los arboles, las montanias, Ios płjaros, el trompo zumbador hablan con fervor animsstico.

$\therefore$ Asf eomo para CaMUs, oto pro ducto telúrico, la presencia ptimordial siempre es la naturaleza, ot mistho para Aktotebas. Ambos, unoen Atgelia yelotto en Perti, son capaces de presentid la violencia, profucto de la injusticia social, que periodictmente estalla, rempiendo berreras de cemento y asfalto en las ciudades.

ARGUEDAs fue capaz de salvarse del infierno viviente de la depresíón escribiendo sobre experiencias tempranas en forma omnidimensional, con la fuerza ayasalladora de la experiencia glopal, Al final, cuando se le cierran sus últimos canales sublimatorios, se suicida.

Mario VARGas Llosa ha estado a la vanguardia de la literatura latinoamericana por más de un cuarto de siglo. Sus cuentos y novelas han sido aclamados en forma universal, culminando con su nominación para el premio Nobel VARGAS LLosA cree que el conocimiento de diferentes niveles de realidad, emerge en la literatura a través de sur transformación por la imaginacion y et uso bef lenguaje: Una novela que trata de pintar la experiencia real en foma objetiva y precisa esta desti nada al fracaso. th cannot succed because the novel was invented, not to transaribe reatity but o transform in: (VABOAS LLOSA, 1991). W: La siguiemte cita, atribufdaa Oscar Witde es acerca de uno de los personajes de BAlzac La muerte de Lucien de Rubempre es el gran drama de mi vidd. Esta cila puede yuxtaponerse a V VARGAS Llosa y FLAUBERT en relación a Emma Bovary. Cuardo un eritico inquisitivo le pregunta a FLAUBERTquín le haservido de modelo para la creáción de su famosa heroma, Flaubert tesponde: Madame Bovary clest woi.

Madame Bovary es teno de los tres "libnos absolutos" del siglo diocinuve, Es uno de los pocos construfdos con un orden simétrico y riguroiso en et que el principio y let fin se uniet formando un chtoto. Madatine Bovaryes unilibto circulas; ino es un libro timear: Esta es lairazón pot la cual el lector deriva en tina sensacion de pode y totalid didmonca antes encontadal despues de su lectura: Aunque serdad /quid Madame Bovary establece la novela realista en Francia, Flaubert rehusó en todo momento a afiliarse con algún movimiento literario.

Al primcipio de sucarrera de escritor, cuando su vida aventurera alcanza sy nadir en los suburbios de Paŕs, V ARGAS LLOSA busca soluciones desesperadas, entre ellas el suicidio. Fué en esta etapa crucial de su vida que redescubre a FLAUBERT y Madame Bovary. Escribe sobre esta experiencia:

Un puñado de personajes literarios han marcado mi vida de manera mas durable que buena parte de lós sereside carne y huesoque he conocido. Aunque es 
verdad que cuando personajes de ficcion y seres humanos son presentes,... -nada tiene tanta vida como el cuerpo que se puede ver, palpar-, la diferencia desaparece cuando ambos tornan a ser pasado, recuerdo, y con ventaja considerable para los primeros sobre los segundos, cuya delicuescencia en la memoria es sin remedio, en tanto que el personaje literario puede ser resucitado indefinidamente, con el mínimo esfuerzo de abrir las páginas del libro y detenerse en las líneas adecuadas..." y luego continúa, "es impagable la ayuda que me prestó en ese período difícil, la historia de Emma, o, mejor dicho la muerte de Emma. Recuerdo haber leído en esos días, con angustiosa avidez, el episodio de su suicidio, haber acudido a esa lectura como otros, en circunstancias parecidas, recurren al cura, la borracherao la morfina, y haber extraído cada vez, de esas páginas desgarradoras, consuelo $\mathrm{y}$ equilibrio, repugnancia del caos, gusto por la vida. El sufrimiento ficticio neutralizaba el que yo vivía. Cada noche, para ayudarme, Emma entraba al desierto castillo de la Huchette y era humillada por Rodolphe; salía al campo donde el dolor y la impotencia la acercaban un instante a la locura; se deslizaba como un duende en la farmacia de Homais, y alli, Justin, la inocencia convertida en secuaz de la muerte, lamirabatragar el arsénico en la penumbra del capharnaum; volvía a su casa y padecía el indecible calvario; el sabor a tinta, la náusea, el frío en los pies, sus estremecimientos, los dedos incrustados en las sábanas, el sudor de su frente, el castañeteo de sus dientes, el extravío de sus ojos, los aullidos, las convulsiones, el vómito de sangre, la lengua que escupe su boca, el estertor final. Cada vez a la tristeza y a la melancolía se mezclaba una curiosa sensación de sosiego y la consecuencia de la lacerante ceremonia era para mi la admiración, el entusiasmo: Emma se mataba para que yo viviera. En otras ocasiones de contrariedad, depresion o simple malhumor he acudido a este remedio y casi siempre con el mismo resultado catártico..."

En la práctica de la clínica psiquiátrica hemos sido testigos de resultados parecidos, especialmente en aquellos pacientes con introspección pasional que prefieren la solitud y la privacidad en la solución de sus problemas existenciales.

\section{ZUSAMMENFASSUNG}

Der Verfasser behauptet, dass eine einzige psychotherapeutische Richtung nicht das lernen vom klassikern in der Literatur und Kunst erklären kann. Erzählungen sind bei Kindern wichting für ein gesundes Geistesleben.

\section{BIBLIOGRAFIA}

1. Arguedas, J. M. (1978): Deep Rivers (F. H. Barraclough, Trans.). University of Texas Ptess, Austin.- 2. Edelman, G. M. (1987): Neural Darwinism: The theory of neuronal group selection, Basic Books, New York.- 3. Foster, E. M. (1927): Aspects of the novel, Penguin, Harmondsworth.- 4. Freud, S. (1959): Collected papers, Vol. 3 (F. Strachey and
J. Strachey, Trans.), Basic Books, New York.- 5. MODEL.L, A. H. (1990): Other times, other realities: Toward a theory of psychoanalytic treatment, Harvard University Press, Cambridge.- 6. VARGas LlosA, M. (1978): La orgía perpetua, Bruguera, Barcelona.- 7. VARGAs Llosa, M. (1991): A writer's reality, Syracuse University Press, Syracuse. 